\title{
BMJ Open Do socioeconomic factors influence breast cancer screening practices among Arab women in Qatar?
}

\author{
Tam Truong Donnelly, ${ }^{1}$ Al-Hareth Al Khater, ${ }^{2}$ Mohamed Ghaith Al Kuwari, ${ }^{3}$ \\ Salha Bujassoum Al-Bader, ${ }^{2}$ Nabila Al-Meer, ${ }^{2}$ Mariam Abdulmalik, ${ }^{4}$ Rajvir Singh, ${ }^{2}$ \\ Sofia Chaudhry, ${ }^{5}$ Tak Fung ${ }^{1}$
}

To cite: Donnelly TT, Al Khater A-H, Al Kuwari MG, et al. Do socioeconomic factors influence breast cancer screening practices among Arab women in Qatar?. BMJ Open 2015;5: e005596. doi:10.1136/ bmjopen-2014-005596

- Prepublication history for this paper is available online. To view these files please visit the journal online (http://dx.doi.org/10.1136/ bmjopen-2014-005596).

Received 1 May 2014

Revised 18 December 2014

Accepted 22 December 2014

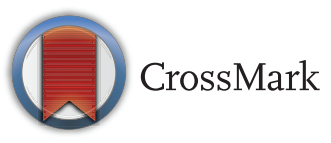

${ }^{1}$ University of Calgary, Calgary, Alberta, Canada ${ }^{2}$ Hamad Medical Corporation, Hamad General Hospital, Doha, Qatar

${ }^{3}$ Aspetar, Doha, Qatar ${ }^{4}$ Department of Primary Health Care, Qatar Supreme Council of Health, Doha,

Qatar

${ }^{5}$ University of Calgary-Qatar, Doha, Qatar

Correspondence to Dr Tam Truong Donnelly; tdonnell@ucalgary.ca

\section{ABSTRACT}

Objectives: Breast cancer incidence rates are rising in Qatar. Although the Qatari government provides subsidised healthcare and screening programmes that reduce cost barriers for residents, breast cancer screening (BCS) practices among women remain low. This study explores the influence of socioeconomic status on BCS among Arab women in Qatar.

Setting: A multicentre, cross-sectional quantitative survey was conducted with 1063 Arab women $(87.5 \%$ response rate) in Qatar from March 2011 to July 2011. Women who were 35 years or older and had lived in Qatar for at least 10 years were recruited from seven primary healthcare centres and women's health clinics in urban and semiurban regions of Qatar. Associations between socioeconomic factors and BCS practice were estimated using $\chi^{2}$ tests and multivariate logistic regression analyses.

Results: Findings indicate that less than one-third of the participants practised BCS appropriately, whereas less than half of the participants were familiar with recent BCS guidelines. Married women and women with higher education and income levels were significantly more likely to be aware of and to practise BCS than women who had lower education and income levels.

Conclusions: Findings indicate low levels of awareness and low participation rates in BCS among Arab women in Qatar. Socioeconomic factors influence these women's participation in BCS activities. The strongest predictors for BCS practice are higher education and higher income levels.

Recommendations: Additional research is needed to explore the impact of economic factors on healthcare seeking behaviours in the Middle Eastern countries that have a high national gross domestic product where healthcare services are free or heavily subsidised by the government; promotion of BCS and intervention strategies in these countries should focus on raising awareness about breast cancer, the cost and benefit of early screening for this disease, particularly among lowincome women.

\section{INTRODUCTION}

In developed countries, breast cancer incidence rates have stabilised or increased

\section{Strengths and limitations of this study}

- Breast cancer is the most common cancer among women in the State of Qatar.

- Women in Qatar are often diagnosed with breast cancer at advanced stages; they are at significant risk for high mortality rate for this disease.

- The information reported in this paper will increase healthcare providers and researchers' understanding of the impact of socioeconomic factors on breast cancer screening in highincome Middle Eastern countries.

- It will offer ways to effectively promote early detection of breast cancer not only for Arabic-speaking women in Qatar, but also for women of similar ethnocultural backgrounds in the Middle East region.

- Non-probability convenience sampling limits the ability to generalise survey results from this study.

slightly in recent years; however, the mortality rate for this disease has been decreasing. ${ }^{1-3}$ In contrast, incidence and mortality rates in Middle Eastern countries are on the rise. ${ }^{4-6}$ It is also found in younger women, and at more developed stages. ${ }^{7}$

Breast cancer is the most common type of cancer among women in the State of Qatar, and screening rates remain low. ${ }^{8}$ In the past few decades, Qatar has experienced substantial development and lifestyle changes driven largely by oil and natural gas wealth. ${ }^{5}$ As overseen by Qatar's Supreme Council of Health, public and private healthcare services in Qatar are modern, subsidised and cost-effective.

Cancer prevalence patterns have changed along with environmental and social changes in Qatar. ${ }^{5-7}$ Although controversies exist regarding the effectiveness of breast cancer screening (BCS), early detection of breast cancer through regular screening activities such as breast self-examination (BSE), clinical 
breast examination (CBE), mammography and improvement of screening quality and treatment have been found to decrease mortality rates of breast cancer. ${ }^{39}$

Significant predictors for CBE and mammography in Qatar and the United Arab Emirates (UAE) are education, urban or semiurban residence, employment and higher socioeconomic status. ${ }^{8}{ }^{10}$ Cost and availability of health insurance are barriers to healthcare in parts of the Middle East; ${ }^{11-13}$ however, these factors do not appear to be barriers where mammography is free, subsidised or covered by insurance, as in Qatar and Saudi Arabia. 81014

Ecological models indicate that an individual's behaviour towards healthcare is influenced by his or her physical environment and by interpersonal and other social determinants of health. ${ }^{15-17}$ To address breast cancer problems and promote the early detection of breast cancer, we investigated how social determinants of health, such as economic and social factors, influence Arab women's healthcare choices and practices. The results related to awareness, knowledge and screening behaviours were reported in a previous publication. ${ }^{18} \mathrm{In}$ this paper, we report (A) participation rates in BCS of Arab women living in Qatar and (B) effects of selected socioeconomic factors on Arab women's awareness and practice of BCS.

\section{METHODS}

Participants were recruited from seven urban hospital settings and community health clinics in Doha (capital of Qatar) and semiurban cities in south and north Qatar. Based on Qatar's 2010 census data, ${ }^{19}$ the study's sample size was calculated using a 95\% confidence level and Cochran's formula for sample size. ${ }^{20}$ Participants were 35 years or older (as previously recommended by Qatar national guidelines for BSE and CBE), had the ability to speak Arabic, were recruited from one of seven designated research sites in Qatar, and had resided in Qatar for at least 10 years. A convenient non-probability sampling technique was used: 1215 self-identified Arab women who met the study's inclusion criteria were invited to participate in the survey, 1063 of them $(40 \%$ more than the required sample size calculation using a margin of error of $3.5 \%$ ) participated in a $30 \mathrm{~min}$ face-to-face interview ( $87.5 \%$ response rate).

Verbal consent for voluntary participation was obtained from each participant. The standard interview protocol and participants' rights were explained to participants and their anonymity and confidentiality was assured.

\section{Questionnaire and data collection}

Data were obtained from in-person interviews using a structured survey questionnaire conducted by female nurses fluent in Arabic and English. Questionnaire items were incorporated from previous peer-reviewed surveys on breast cancer with permission from the authors. $^{21-28}$ Awareness and practice of BCS were defined by recommendations in the most widely disseminated national guidelines. For example, participants were assessed with appropriate BCS practice if they performed BSE monthly, if 35 years or older and had undergone a CBE, or if 40 years or older and had a mammogram within the past 2 years. Forwardtranslations and back-translations of the survey questionnaire into Arabic and English were carried out to ensure lexical equivalence.

\section{Statistical analysis}

Descriptive statistics analyses (mean, SD for interval variables and frequency with percentages for categorical variables) were performed for the study variables where appropriate. $\chi^{2}$ Tests were applied to test for associations between socioeconomic factors and dependent variables (BSE, CBE and mammogram practice). Multicollinearity testing was performed before introducing independent variables into the multivariate analysis. Multivariate logistic regression analyses using the forward stepwise method were used to further assess the association of preselected socioeconomic and demographic factors with binary dependent variables (eg, appropriate practice of BSE, CBE and mammogram). All statistical tests were two-sided with significance established at an $\alpha$ of 0.05 . Data analyses were performed under direct instruction from the researchers and conducted by two senior biostatisticians using SPSS V.20.

\section{RESULTS}

\section{Selected demographic characteristics of participants}

Participants were between the ages of 35-82 years $(\mathrm{M}=44.9, \mathrm{SD}=8.4, \mathrm{n}=1063)$. The majority of the 1063 participants were married $(78.9 \%)$, were Muslim (98.2\%), had children $(84.8 \%)$ and resided in urban areas $(88.7 \%)$. Over half $(52.1 \%)$ of the participants were Qatari citizens; $47.9 \%$ were Qatari residents from the greater Middle Eastern region: $10.9 \%$ were from other Gulf Cooperation Council (GCC) and regional countries (Saudi Arabia, UAE, Kuwait, Oman, Bahrain, Yemen), $16 \%$ were from Levant countries (Syria, Lebanon, Palestine, Jordan), $10.1 \%$ were from North African countries (Egypt, Libya, Tunisia, Algeria, Morocco) and $10.9 \%$ were from other countries (Sudan, Iraq, Iran, Somalia, Mauritania, Pakistan).

Approximately one-third of participants were university-educated, employed and had husbands who were university-educated. Most participants were homemakers $(59.8 \%)$. Of those who reported their annual household income $(54.3 \%)$, approximately three-quarters reported an income of US\$29390 (QAR 107000) or higher (table 1).

\section{Awareness and BCS participation rates}

Previous findings on the same population ${ }^{18}$ indicated that less than half of the participants in the study were aware of BCS recommendations (BSE 28.9\%, CBE 
Table 1 Selected demographic characteristics of participants $(\mathrm{N}=1063)$

\begin{tabular}{|c|c|}
\hline Characteristic & $\begin{array}{l}\text { Number }(\%) \text { of } \\
\text { participants }\end{array}$ \\
\hline \multicolumn{2}{|l|}{ Age (years)* } \\
\hline $35-39$ & $365(34.4)$ \\
\hline $40-49$ & $399(37.6)$ \\
\hline $50+$ & $297(28.0)$ \\
\hline \multicolumn{2}{|l|}{ Nationality } \\
\hline Qatari citizen & $554(52.1)$ \\
\hline Non-Qatari resident & 509 (47.9) \\
\hline Other GCC/peninsular & $116(10.9)$ \\
\hline Levant & $170(16.0)$ \\
\hline North African & $107(10.1)$ \\
\hline Other & $116(10.9)$ \\
\hline \multicolumn{2}{|l|}{ Marital status } \\
\hline Single & $224(21.1)$ \\
\hline Married & 839 (78.9) \\
\hline \multicolumn{2}{|l|}{ Number of children } \\
\hline 0 (none) & $161(15.2)$ \\
\hline$\leq 5$ children & $516(48.5)$ \\
\hline$>5$ children & $386(36.3)$ \\
\hline \multicolumn{2}{|l|}{ Religion } \\
\hline Muslim & 1044 (98.2) \\
\hline Christian & $19(1.8)$ \\
\hline \multicolumn{2}{|l|}{ Living area } \\
\hline Urban & $943(88.7)$ \\
\hline Semiurban & $120(11.3)$ \\
\hline \multicolumn{2}{|l|}{ Education level of participant } \\
\hline$\leq$ Primary/intermediate & $359(33.8)$ \\
\hline Secondary/trade school & 350 (32.9) \\
\hline University & 354 (33.3) \\
\hline \multicolumn{2}{|c|}{ Education level of participant's husband $(n=896)$} \\
\hline$\leq$ Primary/intermediate & $276(30.8)$ \\
\hline Secondary/trade school & $292(32.6)$ \\
\hline University & $328(36.6)$ \\
\hline \multicolumn{2}{|l|}{ Employment status of participant } \\
\hline Employed & $362(34.1)$ \\
\hline Unemployed or homemaker & $701(65.9)$ \\
\hline \multicolumn{2}{|l|}{ Occupation-participant } \\
\hline Unemployed & $75(7.2)$ \\
\hline Management, science, arts & $225(21.5)$ \\
\hline Sales and office & $59(5.6)$ \\
\hline $\begin{array}{l}\text { Services, production, construction, } \\
\text { Transportation, other }\end{array}$ & $62(5.9)$ \\
\hline Homemaker & $626(59.8)$ \\
\hline \multicolumn{2}{|l|}{ Occupation-husband $(n=896) \dagger$} \\
\hline Management, business, science, arts & $305(35.7)$ \\
\hline Services & 105 (12.3) \\
\hline Sales and office & $130(15.2)$ \\
\hline $\begin{array}{l}\text { Construction, production, transportation, } \\
\text { Other }\end{array}$ & $104(12.2)$ \\
\hline Military & $89(10.4)$ \\
\hline Unemployed or retired & $122(14.3)$ \\
\hline \multicolumn{2}{|l|}{ Annual household income } \\
\hline$<$ QAR 107 000/<US\$29 390 & 138 (23.9) \\
\hline QAR 107 000-286 000/ & $274(47.5)$ \\
\hline US\$29 390-US\$78 560 & \\
\hline >QAR 286 000/>US\$78 560 & $165(28.6)$ \\
\hline \multicolumn{2}{|c|}{$\begin{array}{l}{ }^{*} \text { Two participants did not answer this question. } \\
\text { †Forty-one participants did not answer this question. } \\
\text { fFour hundred and eighty-six participants did not answer this } \\
\text { question. } \\
\text { GCC, Gulf Cooperation Council. }\end{array}$} \\
\hline
\end{tabular}

$41.8 \%$, mammography $26.4 \%$ ). Less than one-third of participants practised BCS according to the recommended national guidelines $(13.9 \%$ reported performance of a monthly BSE, 31.3\% had had a CBE within the past 1-2 years, and $26.9 \%$ of participants 40 years of age or older had undergone a mammogram within the past 1-2 years).

\section{Relationship between selected socioeconomic factors and BCS awareness and practice}

Married Qatari resident women from the Levant and North Africa with higher education levels, who also had husbands with higher education levels, or higher annual household incomes were significantly more likely to practise BCS than women of other nationalities, or women who had lower education and income levels. Previous findings ${ }^{18}$ indicated that BCS practice was not associated with nationality when comparing Qatari citizens to Qatari residents. However, further analysis in this paper suggests that BCS practice is significantly associated with nationality when comparing Qatari citizens to Qatari resident subcategories (other GCG, Levant, North African, other).

Participants in the present study were more likely to be aware of BSE if they were employed or had a husband who worked in management. They were more likely to be aware of mammography recommendations if they lived in urban areas or if they (or their husbands) worked in management fields (table 2).

Married women participants with 1-5 children, higher education (participant or husband) or higher income level were more likely to participate in BCS activities than their unmarried peers or counterparts with lower education or income levels. Living area, however, was not significantly related to BCS practice.

Participants who worked in management were more likely to practise BSE than participants who were unemployed or who worked under management, but were less likely to practise BSE if their husbands were unemployed or retired. Although occupation was significantly related to BCS awareness, it was not significantly related to practice of CBE or to mammograms (table 3).

In addition to being less likely to practise BCS than Qatari citizens and participants from the Levant and North Africa, participants from other GCC countries were more likely to be homemakers than women of other nationalities, to have six or more children, and to not have a university education. Their husbands were also less likely to have university degrees, and more likely to work in the military than other nationality groups. Participants from the Levant and North Africa (and their husbands) were more likely to have a university education and work in management occupations than those from other nationality groups. Qatari resident participants from Sudan, Iraq, Iran, Somalia, Mauritania and Pakistan were more likely to report the lowest income levels of all groups, although they did not 


\begin{tabular}{|c|c|c|c|c|c|c|c|c|c|}
\hline \multirow[b]{2}{*}{ Variables } & \multicolumn{3}{|c|}{ BSE awareness } & \multicolumn{3}{|c|}{ CBE awareness } & \multicolumn{3}{|c|}{ Mammogram awareness } \\
\hline & $\begin{array}{l}\text { Yes } \\
\text { n (\%) }\end{array}$ & $\begin{array}{l}\text { No } \\
\text { n (\%) }\end{array}$ & p Value & $\begin{array}{l}\text { Yes } \\
\text { n (\%) }\end{array}$ & $\begin{array}{l}\text { No } \\
\text { n (\%) }\end{array}$ & p Value & $\begin{array}{l}\text { Yes } \\
\text { n (\%) }\end{array}$ & $\begin{array}{l}\text { No } \\
\text { n (\%) }\end{array}$ & p Value \\
\hline Nationality & & & $\chi^{2}(4, N=1063)$ & & & $\chi^{2}(4, N=1063)$ & & & $\chi^{2}(4, N=696)=29.02$ \\
\hline Qatari citizen & $141(25.5)$ & $413(74.5)$ & $=29.37, p<0.001$ & $223(40.3)$ & $331(59.7)$ & $=11.31, p=0.023$ & $77(20.3)$ & $302(79.7)$ & $\mathrm{p}<0.001$ \\
\hline Other GCC resident & $18(15.5)$ & $98(84.5)$ & & $37(31.9)$ & $79(68.1)$ & & 7 (10.6) & 59 (89.4) & \\
\hline Levant resident & $66(38.8)$ & $104(61.2)$ & & $83(48.8)$ & $87(51.2)$ & & 36 (31.3) & 79 (68.7) & \\
\hline North Africa resident & $43(40.2)$ & $64(59.8)$ & & $53(49.5)$ & $54(50.5)$ & & $30(41.7)$ & $42(58.3)$ & \\
\hline Other resident & 39 (33.6) & $77(66.4)$ & & $48(41.4)$ & $68(58.6)$ & & $23(35.9)$ & $41(64.1)$ & \\
\hline Marital status & & & $\chi^{2}(1, N=1063)$ & & & $\chi^{2}(1, N=1063)$ & & & $\chi^{2}(1, N=696)=10.85$ \\
\hline Single & $51(22.8)$ & $173(77.2)$ & $=5.16, p=0.023$ & $63(28.1)$ & $161(71.9)$ & $=21.72, p<0.001$ & $21(14.4)$ & $125(85.6)$ & $\mathrm{p}=0.001$ \\
\hline Married & $256(30.5)$ & $583(69.5)$ & & $381(45.4)$ & $458(54.6)$ & & $152(27.6)$ & $398(72.4)$ & \\
\hline Living area & & & $\chi^{2}(1, N=1063)$ & & & $\chi^{2}(1, N=1063)$ & & & $\chi^{2}(1, N=696)=10.23$ \\
\hline Urban & $274(29.1)$ & 669 (70.9) & $=0.13, p=0.723$ & $386(40.9)$ & $557(59.1)$ & $=2.40, p=0.122$ & $164(26.8)$ & 448 (73.2) & $\mathrm{p}=0.001$ \\
\hline Semiurban & $33(27.5)$ & $87(72.5)$ & & $58(48.3)$ & $62(51.7)$ & & $9(10.7)$ & 75 (89.3) & \\
\hline Education level-participant & & & $\chi^{2}(2, \mathrm{~N}=1063)$ & & & $\chi^{2}(2, N=1063)$ & & & $\chi^{2}(2, \mathrm{~N}=696)=66.00$ \\
\hline$\leq$ Primary/intermediate & $42(11.7)$ & $317(88.3)$ & $=90.99, p<0.001$ & $126(35.1)$ & $233(64.9)$ & $=13.34, p=0.001$ & 33 (11.2) & $261(88.8)$ & $\mathrm{p}<0.001$ \\
\hline Secondary/trade & $110(31.4)$ & $240(68.6)$ & & $146(41.7)$ & $204(58.3)$ & & $59(27.1)$ & 159 (72.9) & \\
\hline University & $155(43.8)$ & $199(56.2)$ & & $172(48.6)$ & $182(51.4)$ & & $81(44.0)$ & $103(56.0)$ & \\
\hline Education level-husband & & & $\chi^{2}(2, N=896)$ & & & $\chi^{2}(2, N=896)$ & & & $\chi^{2}(2, N=604)=36.61$ \\
\hline$\leq$ Primary/intermediate & $35(12.7)$ & $241(87.3)$ & $=57.73, p<0.001$ & $90(32.6)$ & $186(67.4)$ & $=21.37, p<0.001$ & $27(12.4)$ & $190(87.6)$ & $\mathrm{p}<0.001$ \\
\hline Secondary & 97 (33.2) & $195(66.8)$ & & $139(47.6)$ & $153(52.4)$ & & $51(27.1)$ & $137(72.9)$ & \\
\hline University & $132(40.2)$ & $196(59.8)$ & & $165(50.3)$ & $163(49.7)$ & & $76(38.2)$ & $123(61.8)$ & \\
\hline Employment status-participant & & & $\chi^{2}(1, N=1063)$ & & & $\chi^{2}(1, N=1063)$ & & & $\chi^{2}(1, N=696)=7.95$ \\
\hline Employed & $144(39.8)$ & $218(60.2)$ & $=31.74, p<0.001$ & $158(43.6)$ & $204(56.4)$ & $=0.80, p=0.372$ & $61(32.4)$ & $127(67.6)$ & $p=0.005$ \\
\hline Unemployed & $163(23.3)$ & $538(76.7)$ & & $286(40.8)$ & 415 (59.2) & & $112(22.0)$ & $396(78.0)$ & \\
\hline Occupation-participant & & & $\chi^{2}(4, N=1047)$ & & & $\chi^{2}(4, N=1047)$ & & & $\chi^{2}(4, N=686)=28.43$ \\
\hline Unemployed & 25 (33.3) & $50(66.7)$ & $=39.09, p<0.001$ & $41(54.7)$ & $34(45.3)$ & $=9.41, p=0.052$ & $20(32.8)$ & $41(67.2)$ & $\mathrm{p}<0.001$ \\
\hline Management, science, arts & $95(42.2)$ & $130(57.8)$ & & $94(41.8)$ & $131(58.2)$ & & $45(44.6)$ & $56(55.4)$ & \\
\hline Sales and office & $20(33.9)$ & $39(66.1)$ & & $24(40.7)$ & $35(59.3)$ & & $7(18.9)$ & $30(81.1)$ & \\
\hline Services, production, other & $25(40.3)$ & $37(59.7)$ & & $32(51.6)$ & $30(48.4)$ & & $8(20.0)$ & $32(80.0)$ & \\
\hline Homemaker & $138(22.0)$ & $488(78.0)$ & & $245(39.1)$ & $381(60.9)$ & & $92(20.6)$ & $355(79.4)$ & \\
\hline Occupation-husband & & & $\chi^{2}(5, N=855)$ & & & $\chi^{2}(5, N=855)=7.60$ & & & $\chi^{2}(5, N=563)=16.83$ \\
\hline Unemployed or retired & $20(16.4)$ & $102(83.6)$ & $=22.24, p<0.001$ & 45 (36.9) & $77(63.1)$ & $p=0.180$ & $18(16.1)$ & 94 (83.9) & $p=0.005$ \\
\hline Management, science, arts & $112(36.7)$ & $193(63.3)$ & & $151(49.5)$ & $154(50.5)$ & & 65 (35.9) & $116(64.1)$ & \\
\hline Service & $39(37.1)$ & 66 (62.9) & & $44(41.9)$ & $61(58.1)$ & & $12(21.4)$ & $44(78.6)$ & \\
\hline Sales and office & $39(30.0)$ & $91(70.0)$ & & $62(47.7)$ & 68 (52.3) & & 29 (32.2) & $61(67.8)$ & \\
\hline Production, other & $24(23.1)$ & $80(76.9)$ & & $42(40.4)$ & $62(59.6)$ & & $19(26.0)$ & $54(74.0)$ & \\
\hline Military & $25(28.1)$ & $64(71.9)$ & & $38(42.7)$ & $51(57.3)$ & & $11(21.6)$ & $40(78.4)$ & \\
\hline Annual household income & & & $\chi^{2}(2, N=577)$ & & & $\chi^{2}(2, N=577)$ & & & $\chi^{2}(2, N=355)=14.48$ \\
\hline$<$ US\$29 390 & $31(22.5)$ & $107(77.5)$ & $=12.47, p=0.002$ & $47(34.1)$ & $91(65.9)$ & $=14.33, p=0.001$ & $13(14.4)$ & $77(85.6)$ & $\mathrm{p}=0.001$ \\
\hline US\$29 390-US\$78 560 & 72 (26.3) & $202(73.7)$ & & $123(44.9)$ & $151(55.1)$ & & $45(26.8)$ & $123(73.2)$ & \\
\hline$>$ US\$78 560 & $65(39.4)$ & $100(60.6)$ & & $92(55.8)$ & $73(44.2)$ & & $38(39.2)$ & $59(60.8)$ & \\
\hline
\end{tabular}

BCS, breast cancer screening; BSE, breast self-examination; CBE, clinical breast examination; GCC, Gulf Cooperation Council. 


\begin{tabular}{|c|c|c|c|c|c|c|c|c|c|}
\hline \multirow[b]{2}{*}{ Variables } & \multicolumn{3}{|c|}{ BSE practice } & \multicolumn{3}{|c|}{ CBE practice } & \multicolumn{3}{|c|}{ Mammogram practice } \\
\hline & $\begin{array}{l}\text { Yes } \\
\text { n (\%) }\end{array}$ & $\begin{array}{l}\text { No } \\
\text { n (\%) }\end{array}$ & p Value & $\begin{array}{l}\text { Yes } \\
\text { n (\%) }\end{array}$ & $\begin{array}{l}\text { No } \\
\mathrm{n}(\%)\end{array}$ & p Value & $\begin{array}{l}\text { Yes } \\
\text { n (\%) }\end{array}$ & $\begin{array}{l}\text { No } \\
\text { n (\%) }\end{array}$ & p Value \\
\hline Nationality & & & $\chi^{2}(4, N=1063)=15.02$ & & & $\chi^{2}(4, N=1063)=10.30$ & & & $\chi^{2}(4, N=695)=9.70$ \\
\hline Qatari citizen & $69(12.5)$ & $485(87.5)$ & $p=0.005$ & $163(29.4)$ & $391(70.6)$ & $p=0.036$ & $98(25.9)$ & $280(74.1)$ & $p=0.046$ \\
\hline Other GCC resident & $7(6.0)$ & $109(94.0)$ & & $29(25.0)$ & $87(75.0)$ & & $9(13.6)$ & $57(86.4)$ & \\
\hline Levant resident & $31(18.2)$ & $139(81.8)$ & & 67 (39.4) & $103(60.6)$ & & 39 (33.9) & $76(66.1)$ & \\
\hline North Africa resident & $23(21.5)$ & $84(78.5)$ & & $40(37.4)$ & 67 (62.6) & & $22(30.6)$ & 50 (64.9) & \\
\hline Other resident & $18(15.5)$ & $98(84.5)$ & & 34 (29.3) & $82(70.7)$ & & 19 (29.7) & $45(70.3)$ & \\
\hline Marital status & & & $\chi^{2}(1, N=1063)=0.23$ & & & $\chi^{2}(1, N=1063)=18.01$ & & & $\chi^{2}(1, N=695)=4.44$ \\
\hline Single & 29 (12.9) & $195(87.1)$ & $p=0.635$ & $44(19.6)$ & $180(80.4)$ & $p<0.001$ & $29(20.0)$ & $116(80.0)$ & $\mathrm{p}=0.035$ \\
\hline Married & 119 (14.2) & $720(85.8)$ & & $289(34.4)$ & $550(65.6)$ & & $158(28.7)$ & $392(71.3)$ & \\
\hline Number of children & & & $\chi^{2}(2, N=1063)=31.31$ & & & $\chi^{2}(2, N=1063)=10.90$ & & & $\chi^{2}(2, N=695)=6.99$ \\
\hline 0 (none) & $26(16.1)$ & $135(83.9)$ & $\mathrm{p}=0.001$ & $35(21.7)$ & $126(78.3)$ & $\mathrm{p}=0.004$ & $16(25.8)$ & $46(74.2)$ & $\mathrm{p}=0.030$ \\
\hline$\leq 5$ & $88(17.1)$ & 428 (82.9) & & $182(35.3)$ & $334(45.8)$ & & 96 (31.9) & $205(68.1)$ & \\
\hline$>5$ & $34(8.8)$ & $352(91.2)$ & & $116(30.1)$ & $270(69.9)$ & & $75(22.6)$ & $257(77.4)$ & \\
\hline Living area & & & $\chi^{2}(1, N=1063)=2.55$ & & & $\chi^{2}(1, N=1063)=0.29$ & & & $\chi^{2}(1, N=695)=3.00$ \\
\hline Urban & $137(14.5)$ & $806(85.5)$ & $p=0.110$ & $298(31.6)$ & $645(68.4)$ & $p=0.588$ & $171(28.0)$ & $440(72.0)$ & $p=0.083$ \\
\hline Semiurban & $11(9.2)$ & $109(90.8)$ & & 35 (29.2) & $85(70.8)$ & & $16(19.0)$ & $68(81.0)$ & \\
\hline Education level-participant & & & $\chi^{2}(2, N=1063)=30.13$ & & & $\chi^{2}(2, N=1063)=12.58$ & & & $\chi^{2}(2, N=695)=13.99$, \\
\hline$\leq$ Primary/intermediate & $24(6.7)$ & $335(93.3)$ & $\mathrm{p}<0.001$ & $89(24.8)$ & $270(75.2)$ & $p=0.002$ & $60(20.4)$ & $234(79.6)$ & $p=0.001$ \\
\hline Secondary/trade & $50(14.3)$ & $300(85.7)$ & & $113(32.3)$ & $237(67.7)$ & & $61(28.1)$ & 156 (71.9) & \\
\hline University & $74(20.9)$ & $280(79.1)$ & & $131(37.0)$ & $223(63.0)$ & & $66(35.9)$ & $118(64.1)$ & \\
\hline Education level-husband & & & $\chi^{2}(2, N=896)=35.22$ & & & $\chi^{2}(2, N=896)=16.24$ & & & $\chi^{2}(2, N=604)=14.06$ \\
\hline$\leq$ Primary/intermediate & $12(4.3)$ & $264(95.7)$ & $\mathrm{p}<0.001$ & $65(23.6)$ & $211(76.4)$ & $p<0.001$ & $41(18.9)$ & $176(81.1)$ & $\mathrm{p}=0.001$ \\
\hline Secondary & $44(15.1)$ & $248(84.9)$ & & $108(37.0)$ & $184(63.0)$ & & $53(28.2)$ & $135(71.8)$ & \\
\hline University & $69(21.0)$ & $259(79.0)$ & & $123(37.5)$ & $205(62.5)$ & & $70(35.2)$ & $129(64.8)$ & \\
\hline Employment status-participant & & & $\chi^{2}(1, N=1063)=14.83$ & & & $\chi^{2}(1, N=1063)=2.19$ & & & $\chi^{2}(1, N=695)=1.53$ \\
\hline Employed & $71(19.6)$ & $291(80.4)$ & $p<0.001$ & $124(34.4)$ & $238(65.7)$ & $\mathrm{p}=0.139$ & $57(30.3)$ & $131(69.7)$ & $\mathrm{p}=0.217$ \\
\hline Unemployed & $77(11.0)$ & $624(89.0)$ & & $209(29.8)$ & 492 (70.2) & & $130(25.6)$ & $377(74.4)$ & \\
\hline Occupation-participant & & & $\chi^{2}(4, N=1047)=29.87$ & & & $\chi^{2}(4, N=1047)=3.97$ & & & $\chi^{2}(4, N=685)=4.90$ \\
\hline Unemployed & $5(6.7)$ & $70(93.3)$ & $\mathrm{p}<0.001$ & $27(36.0)$ & $48(64.0)$ & $\mathrm{p}=0.410$ & $18(29.5)$ & $43(70.5)$ & $p=0.298$ \\
\hline Management, science, arts & $55(24.4)$ & $170(75.6)$ & & 74 (32.9) & $151(67.1)$ & & $33(32.7)$ & $68(67.3)$ & \\
\hline Sales and office & $4(6.8)$ & 55 (93.2) & & $22(37.3)$ & $37(62.7)$ & & $13(35.1)$ & $24(64.9)$ & \\
\hline Services, production, other & $10(16.1)$ & 52 (83.9) & & $22(35.5)$ & $40(64.5)$ & & $8(20.0)$ & $32(80.0)$ & \\
\hline Homemaker & $72(11.5)$ & $554(88.5)$ & & $182(29.1)$ & 444 (70.9) & & $112(25.1)$ & $334(74.9)$ & \\
\hline Occupation—husband & & & $\chi^{2}(5, N=855)=16.17$ & & & $\chi^{2}(5, N=855)=9.81$ & & & $\chi^{2}(5, N=563)=8.28$ \\
\hline Management & $57(18.7)$ & $248(81.3)$ & $p=0.006$ & $122(40.0)$ & $183(60.0)$ & $\mathrm{p}=0.081$ & $62(34.3)$ & $119(65.7)$ & $\mathrm{p}=0.141$ \\
\hline Service & $19(18.1)$ & $86(81.9)$ & & 38 (36.2) & $67(63.8)$ & & $17(30.4)$ & $39(69.6)$ & \\
\hline Sales and office & $12(9.2)$ & $118(90.8)$ & & 41 (31.5) & $89(68.5)$ & & $28(31.1)$ & 62 (68.9) & \\
\hline Production, other & $11(10.6)$ & $93(89.4)$ & & $31(29.8)$ & $73(70.2)$ & & $16(21.9)$ & $57(78.1)$ & \\
\hline Military & $14(15.7)$ & $75(84.3)$ & & $26(29.2)$ & $63(70.8)$ & & $12(23.5)$ & $39(76.5)$ & \\
\hline Unemployed/retired & $8(6.6)$ & $114(93.4)$ & & $33(27.0)$ & $89(73.0)$ & & $24(21.4)$ & $88(78.6)$ & \\
\hline Annual household income & & & $\chi^{2}(2, N=577)=7.39$ & & & $\chi^{2}(2, N=577)=23.44$ & & & $\chi^{2}(2, N=354)=25.71$, \\
\hline$<$ US\$29 390 & $13(9.4)$ & $125(90.6)$ & $\mathrm{p}=0.025$ & 27 (19.6) & $111(80.4)$ & $\mathrm{p}<0.001$ & $11(12.2)$ & $79(87.8)$ & $p<0.001$ \\
\hline US\$29 390-US\$78 560 & $36(13.1)$ & $238(86.9)$ & & $98(35.8)$ & $176(64.2)$ & & 53 (31.7) & $114(68.3)$ & \\
\hline$>$ US\$78 560 & $33(20.0)$ & $132(80.0)$ & & $76(46.1)$ & 89 (53.9) & & $45(46.4)$ & $52(53.6)$ & \\
\hline
\end{tabular}


Table 4 Association between selected factors and BCS practice (significant at $\alpha=0.05$ level)

\begin{tabular}{|c|c|c|c|c|}
\hline & & & Adjusted OR (95\% Cl) & p Value \\
\hline \multicolumn{5}{|c|}{ Predictors of BSE practice $(n=445)$} \\
\hline \multicolumn{3}{|c|}{$\begin{array}{l}\left.\text { Education level-husband (Wald } \chi^{2}(2)=6.22\right) \\
\leq \text { Primary/intermediate }\end{array}$} & & 0.045 \\
\hline \multicolumn{3}{|c|}{ Secondary } & $3.11(1.21$ to 8.00$)$ & 0.019 \\
\hline \multirow{2}{*}{\multicolumn{3}{|c|}{$\begin{array}{l}\text { University } \\
\left.\text { Occupation-participant (Wald } \chi^{2}(4)=9.93\right)\end{array}$}} & 3.05 (1.22 to 7.63$)$ & 0.018 \\
\hline & & & & 0.042 \\
\hline \multicolumn{3}{|c|}{ Unemployed } & 1.00 & \\
\hline \multicolumn{3}{|c|}{ Management, science, arts } & 3.51 (0.99 to 12.47$)$ & 0.053 \\
\hline \multicolumn{3}{|l|}{ Sales and office } & $1.66(0.30$ to 9.11$)$ & 0.561 \\
\hline \multicolumn{3}{|c|}{ Services, production, other } & $3.43(0.73$ to 16.23$)$ & 0.120 \\
\hline \multicolumn{3}{|c|}{ Homemaker } & $1.51(0.43$ to 5.29$)$ & 0.520 \\
\hline \multicolumn{5}{|l|}{ Model summary } \\
\hline-2 Log likelihood & Cox and Snell $\mathbf{R}^{2}$ & Nagelkerke $\mathbf{R}^{2}$ & & \\
\hline 343.92 & 0.042 & 0.075 & & \\
\hline \multicolumn{5}{|c|}{ Predictors of CBE Practice $(n=445)$} \\
\hline \multicolumn{3}{|c|}{ Annual Income (Wald $\left.\chi^{2}(2)=11.90\right)$} & & 0.003 \\
\hline \multicolumn{3}{|l|}{$<$ US\$29 390} & 1.00 & \\
\hline \multicolumn{3}{|c|}{ US\$29 390-US\$78 560} & 1.89 (1.07 to 3.36$)$ & 0.029 \\
\hline \multirow{2}{*}{\multicolumn{3}{|c|}{$\frac{>\text { US } \$ 78560}{\text { Model summary }}$}} & 2.84 (1.56 to 5.15$)$ & 0.001 \\
\hline & & & & \\
\hline -2 Log likelihood & Cox and Snell $R^{2}$ & Nagelkerke $\mathbf{R}^{2}$ & & \\
\hline \multicolumn{3}{|l|}{574.21} & & \\
\hline \multicolumn{4}{|c|}{ Predictors of Mammogram Practice (40+ years, $n=267)$} & \\
\hline \multirow{2}{*}{\multicolumn{3}{|c|}{$\begin{array}{l}\left.\text { Annual Income (Wald } \chi^{2}(2)=11.52\right) \\
<\text { US } \$ 29390\end{array}$}} & & 0.003 \\
\hline & & & 1.00 & \\
\hline \multicolumn{3}{|c|}{ US\$29 390-US\$78 560} & 2.67 (1.11 to 6.45$)$ & 0.029 \\
\hline \multicolumn{3}{|l|}{$>$ US\$78 560} & $4.63(1.87$ to 11.47$)$ & 0.001 \\
\hline \multicolumn{5}{|l|}{ Model summary } \\
\hline -2 Log likelihood & Cox and Snell $R^{2}$ & Nagelkerke $\mathbf{R}^{2}$ & & \\
\hline 320.91 & 0.048 & 0.067 & & \\
\hline
\end{tabular}

have the lowest BCS awareness or practice levels. Qatari citizen participants reported the highest incomes of all groups, even though more of their husbands were unemployed or retired.

\section{Multivariate analysis of socioeconomic factors associated with BCS practice}

Table 4 reports socioeconomic factors that might predict BCS activity among participants based on a forward stepwise multivariate logistic regression analysis. Selected independent variables were nationality, living area, education, income level and occupation group: Although the Nagelkerke $R^{2}$ values indicate that the model does not fit the data well, the selected independent variables -income, husband's education level and participant's occupation-significantly predict BCS activity. Annual income significantly predicted CBE and mammogram practice. Participants in the mid to highest annual household income level groups had higher odds of having CBEs or mammograms than those in the lowest income group. Those with the highest reported income levels had over four times the odds of having mammograms than those with the lowest reported income levels. Although nationality was significantly associated with BCS practice, nationality, education level and living area were not found to be predictors of participants' BCS practice.

\section{DISCUSSION}

Results indicate that most Arabic women living in Qatar are not aware of, and they do not practise BCS according to national guidelines. ${ }^{18}$ Further analysis of data indicate that nationality and income levels are significantly related to participants' BCS awareness and practice but are not significant predictors of the women's BSE practice. Consistent with previous research, higher education levels are associated with higher BCS awareness and practice. $^{8} 1029$

The State of Qatar has the highest gross domestic product in the world and provides free or subsidised gender-appropriate healthcare services to citizens and 
residents in state-of-the-art hospitals. It is often assumed that minimal healthcare costs to patients/clients and gender-appropriate healthcare services would increase health-seeking behaviours in countries like Qatar. ${ }^{8} 1014$ However, the low BCS rates in Qatar indicate that more complex factors may be at work. Previous studies done in countries where healthcare is also subsidised by the government, such as Canada, indicate that despite healthcare costs being heavily subsidised by the government, multiple barriers such as lower education levels, language or transportation problems, multiple role problems and limited social support networks might contribute to lower accessibility to BCS for lower-income women. $^{2129}$

Participants from other GCC countries living in Qatar had lower education levels than other women's groups. They had the highest number of children, thus having more domestic responsibility than other women in this study. In addition to having lower awareness of BCS and its benefits, it is likely that these women may not be fully aware of the subsidised healthcare costs and modern equipment available in Qatar because they have come from different countries with different healthcare systems. Compounded with the lower education levels of their husbands, these factors might contribute to a constrained ability to participate in BCS programmes. Thus, these women are at higher risk of having breast cancer diagnosed at later stages of the disease.

A cross-sectional study conducted in nearby Saudi Arabia revealed that $90 \%$ of male participants did not know that mammography can provide early detection of breast cancer. ${ }^{30}$ Male relatives can influence healthseeking behaviours in traditional societies, ${ }^{31}$ as our results indicate (higher educated husbands were associated with a higher BCS uptake). Thus, more efforts to promote awareness among men of breast cancer and the benefits of screening for this disease should be considered. It has been suggested that if women become more aware of the benefits of BCS, the effects of other compounding barriers may be lessened. ${ }^{29}$ However, raising awareness might not be sufficient; further investigation of additional sociocultural barriers, such as personal and healthcare system barriers and cultural beliefs, must be considered among women in higher-risk groups.

In this study, women from the Levant or North Africa or women with higher education levels were significantly more aware of and practised more BCS activities than Qatari citizen participants. It is generally assumed that higher income is associated with higher education and higher health-seeking behaviour. ${ }^{21}$ However, Qatari citizen women reported the highest annual incomes, despite having a lower BCS awareness and uptake compared with women from the Levant or North Africa. This can be explained by the fact that the Qatari government provides cost-of-living stipends for all Qatari citizens. Furthermore, health professionals in cancer research and screening centres have observed that while Qatari citizen women might not have financial barriers, they often shy away from screening due to anonymity issues and fear of discovering cancer. ${ }^{8} 10$

Further research is needed to explore sociocultural and economic variances among Arab women that may be specific to each population. Without a proper understanding of the influence of these sociocultural and economic factors on health-seeking or health-avoiding behaviours, the effectiveness and sustainability of intervention programmes cannot be achieved.

Owing to the challenges of reaching the study population, convenience sampling was used. This might limit the ability to generalise survey results from this study. However, randomly selected times were chosen for the face-to-face interviews, and attempts were made to approach all potential respondents in every interview location that would reduce this bias. The procedures resulted in a response rate of over $87.5 \%$. Since $45.7 \%$ of the women interviewed did not volunteer their income levels, our sample size was reduced when conducting logistic regression analyses $(n=445)$. Also, data collected from self-reported face-to-face interviews might be subject to recall or social-desirability response bias.

\section{CONCLUSION}

Breast cancer incidence rates in the Middle East are rising and mortality rates are disproportionally high compared with North American and European countries. ${ }^{5}$ As this study's findings indicate, socioeconomic factors do influence BCS practices among women living in Qatar. Thus, a further reduction in costs or free services for lower-income women, and more accessible mammogram facilities in all regions of Qatar, could facilitate women's utilisation of BCS activities. To increase women's participation rates in BCS activities, non-opportunistic population-based national screening programmes are also urgently needed in countries like Qatar. ${ }^{32}$ Meanwhile, healthcare professionals must be at the forefront of raising awareness of both female and male patients, regardless of nationality, education or socioeconomic status, of the benefits and availability of early cancer detection and BCS services in Qatar. A multilevel approach to raising awareness about the cost and benefit of BCS among at-risk low-income women and the general population should include the involvement of allied healthcare professionals, local health centres, national mass-media campaigns, male relatives, breast cancer survivors, and religious and community leaders.

Despite the low screening rates, it is encouraging to note that Arab women are eager to learn more about $\mathrm{BCS}$, and allied health professionals are willing to discuss BCS with patients. ${ }^{13} 18{ }^{33-37}$ Studies of women's health practices often focus on examination of differences in health beliefs and cultural values of the women. Although it is important to appreciate the effect of cultural assumptions on healthcare practice, it is also imperative to examine the social and economic dimensions of women's healthcare experience. Future research 
should investigate additional factors that younger generations of women living in rapidly changing societies like Qatar might face, including the interplay of modernity and cultural expectations, private versus public healthcare facilities, and increasingly higher income, employment and education levels among Arab women. Knowledge gained from this study will benefit countries with sociodemographics similar to Qatar throughout the Middle East.

Acknowledgements The authors are grateful to all the women who participated in this research and to the Qatar National Research Fund which provided us with funding (National Priorities Research Program -NPRP 09-261-3-059) to conduct this study. They give special thanks to staff at the Hamad Medical Corporation (Hamad General Hospital, Women Hospital) and the Qatar Primary Health Care, Community Health Clinics who helped us recruit research participants. They also thank the research assistants Roqaia Ahmad Dorri, Shima Sharara, Aisha Al-Ali, Aisha Al-Khayren, Asma Albulushi, Asma Rehman, Fadi Al-Massri, Khadra Yassin, Salah Hmaid, Yasser Sami, Zeinab Idris, Noora Rashid Al Enazi and Nahrida Nazir Khiyal Meer, and their former project managers Floor Christie de Jong and SC.

Contributors TTD contributed to the conception and design of the study and the acquisition, analysis and interpretation of data, drafted the manuscript, and gave final approval of the manuscript version submitted for publication. A-HAK, SBA-B and NA-M contributed to the conception and design of the study and the acquisition of data, revised the manuscript, and gave final approval of the manuscript version submitted for publication. MGAK and MA contributed to the conception and design of the study and the acquisition of data, reviewed the manuscript critically for content, and gave final approval of the manuscript version submitted for publication. RS contributed to the conception and design of the study and the acquisition, analysis and interpretation of data, revised the manuscript, and gave final approval of the manuscript version submitted for publication. SC contributed to the analysis and interpretation of data, drafted the manuscript, and gave final approval of the manuscript version submitted for publication. TF contributed to the analysis and interpretation of data, revised the manuscript, and gave final approval of the manuscript version submitted for publication.

Funding This study was made possible by a grant from the Qatar National Research Fund under its National Priorities Research Program (NPRP 09-2613-059). Its contents are solely the responsibility of the authors and do not necessarily represent the official views of the Qatar National Research Fund.

Competing interests None.

Ethics approval Ethics approval for this research study was obtained from the Hamad Medical Corporation Research Committee (Ethics Approval Reference No: RC/1744/2010), the Qatar Supreme Council of Health (Ethics Assurance No: SCH-AUCQ-050), and the University of Calgary's Conjoint Health Research Ethics Board (Ethics ID: E-23551).

Provenance and peer review Not commissioned; externally peer reviewed.

Data sharing statement No additional data are available.

Open Access This is an Open Access article distributed in accordance with the Creative Commons Attribution Non Commercial (CC BY-NC 4.0) license, which permits others to distribute, remix, adapt, build upon this work noncommercially, and license their derivative works on different terms, provided the original work is properly cited and the use is non-commercial. See: http:// creativecommons.org/licenses/by-nc/4.0/

\section{REFERENCES}

1. World Health Organization (WHO). Breast cancer: prevention and control. http://www.who.int/cancer/detection/breastcancer/en/index. html (accessed 6 May 2012).

2. International Agency for Research Cancer (IARC), WHO. The GLOBOCAN project fact sheets. http://globocan.iarc.fr/ (accessed 6 May 2012).
3. Mai V, Sullivan T, Chiarelli AM. Breast cancer screening program in Canada: successes and challenges. Salud Publica Mex 2009;51 (Suppl 2):s228-35.

4. Forouzanfar $\mathrm{MH}$, Foreman $\mathrm{KJ}$, Delossantos $\mathrm{AM}$, et al. Breast and cervical cancer in 187 countries between 1980 and 2010: a systematic analysis. Lancet 2011;378:1461-84.

5. Bener A, Ayub H, Kakil R, et al. Patterns of cancer incidence among the population of Qatar: a worldwide comparative study. Asian Pac J Cancer Prev 2008;9:19-24.

6. Azaiza F, Cohen M. Health beliefs and rates of breast cancer screening among Arab women. J Womens Health (Larchmt) 2006;15:520-30.

7. Tarabeia J, Baron-Epel O, Barchana M, et al. A comparison of trends in incidence and mortality rates of breast cancer, incidence to mortality ratio and stage at diagnosis between Arab and Jewish women in Israel, 1979-2002. Eur J Cancer Prev 2007;16:36-42.

8. Bener A, El Ayoubi HR, Moore MA, et al. Do we need to maximise the breast cancer screening awareness? Experience with an endogamous society with high fertility. Asian Pac J Cancer Prev 2009;10:599-604.

9. Tabar L, Vitak B, Chen $\mathrm{HH}$, et al. The Swedish two-county trial twenty years later. Updated mortality results and new insights from long-term follow-up. Radiol Clin North Am 2000;38:625-51.

10. Bener A, Alwash R, Miller CJ, et al. Knowledge, attitudes, and practices related to breast cancer screening: a survey of Arabic women. J Cancer Educ 2001;16:215-20.

11. Alkhasawneh IM, Akhu-Zaheya LM, Suleiman SM. Jordanian nurses' knowledge and practice of breast self-examination. J Adv Nurs 2009;65:412-16.

12. Lamyian M, Hydarnia A, Ahmadi F, et al. Barriers to and factors facilitating breast cancer screening among Iranian women: a qualitative study. East Mediterr Health J 2007;13:1160-9.

13. Petro-Nustas WI. Factors associated with mammography utilization among Jordanian women. J Transcult Nurs 2001;12:284-91.

14. Amin TT, Al Mulhim AR, Al Meqihwi A. Breast cancer knowledge, risk factors and screening among adult Saudi women in a primary health care setting. Asian Pac J Cancer Prev 2009;10:133-8.

15. Green LW, Richard L, Potvin L. Ecological foundations of health promotion. Am J Health Promot 1996;10:270-81.

16. Hamilton N, Bhatti T. Population health promotion: an integrated model of population health and health promotion. Ottawa: Health Promotion Development Division, Health Canada, 1996.

17. Vollman A, Anderson ET, McFarlane J. Canadian community as partner 2nd edn. Philadelphia, PA: Lippincott Williams \& Wilkins, 2008.

18. Donnelly TT, Al-Khater A, Al-Bader SB, et al. Breast cancer screening among Arabic women living in the State of Qatar: awareness, knowledge, and participation in screening activities. Avicenna 2012. http://www.qscience.com/doi/abs/10.5339/avi.2012.2

19. Qatar Census. Final results of census 2010. Population. http://www. qsa.gov.qa/QatarCensus/Populations.aspx (accessed 7 May 2012)

20. Cochran GG, Fletcher J, Krapf JH, et al. The determination of developmental norms for a pre-school rural population. J Am Med Womens Assoc 1977:32:123-36.

21. Donnelly TT, McKellin W, Hislop G, et al. Socioeconomic influences on Vietnamese-Canadian women's breast and cervical cancer prevention practices: a social determinant's perspective. Soc Work Public Health 2009;24:454-76.

22. Donnelly TT, McKellin W. Vietnamese Canadian women's breast cancer and cervical cancer screening: the influence of gendered roles and expectations. Anthropol Aging Q 2008;29:76-88.

23. Donnelly TT, McKellin W. Keeping healthy! Whose responsibility is it anyway? Vietnamese Canadian women and their healthcare providers' perspectives. Nurs Inq 2007;14:2-12.

24. Donnelly TT. The health-care practices of Vietnamese-Canadian women: cultural influences on breast and cervical cancer screening. Can J Nurs Res 2006;38:82-101.

25. McPhee SJ, Stewart S, Brock KC, et al. Factors associated with breast and cervical cancer screening practices among Vietnamese American women. Cancer Detect Prev 1997;21:510-21.

26. McPhee SJ, Nguyen TT. Cancer, cancer risk factors, and community-based cancer control trials in Vietnamese Americans. Asian Am Pac Isl J Health 2000;8:18-31.

27. McPhee SJ, Nguyen TT, Shema SJ, et al. Validation of recall of breast and cervical cancer screening by women in an ethnically diverse population. Prev Med 2002;35:463-73.

28. Cheek J, Fuller J, Gilchrist S, et al. Vietnamese women and pap smears: issues in promotion. Aust N Z J Public Health 1999;23:72-6.

29. Soskolne V, Marie S, Manor O. Beliefs, recommendations and intentions are important explanatory factors of mammography screening behavior among Muslim Arab women in Israel. Health Educ Res 2007;22:665-76. 
30. Al-Amoudi SM, Abduljabbar HS. Men's knowledge and attitude towards breast cancer in Saudi Arabia. A cross-sectional study. Saudi Med J 2012;33:547-50.

31. Sabih WK, Taher JA, El Jabari C, et al. Barriers to breast cancer screening and treatment among women in Emirate of Abu Dhabi. Ethn Dis 2012;22:148-54.

32. Donnelly TT, Hwang J. Breast cancer screening interventions for Arabic women: a literature review. J Immigr Minor Health 2013. http://link.springer.com.ezproxy.lib.ucalgary.ca/article/10.1007/ s10903-013-9902-9

33. El Hajj MS, Hamid Y. Breast cancer health promotion in Qatar: a survey of community pharmacists' interests and needs. Int J Clin Pharm 2011;33:70-9.
34. Ahmed BA. Awareness and practice of breast cancer and breast-self examination among university students in Yemen. Asian Pac J Cancer Prev 2010;11:101-5.

35. Ravichandran K, Al-Hamdan NA, Mohamed G. Knowledge, attitude, and behavior among Saudis toward cancer preventive practice. J Family Community Med 2011;18:135-42.

36. Al-Qattan M, Al Saleh K, Al-Musallam S, et al. Knowledge and factors affecting breast self-examination among Kuwaiti women. Kuwait Med J 2008;40:103-10.

37. Milaat WA. Knowledge of secondary-school female students on breast cancer and breast self-examination in Jeddah, Saudi Arabia. East Mediterr Health J 2000;6:338-44. 